\title{
Design and Implementation of Intra band Contiguous Component Carriers on LTE-A
}

\author{
A. Z. Yonis \\ Dept. of Communication Eng. \\ College of Electronics Eng. \\ University of Mosul, Iraq
}

\author{
M. F. L. Abdullah \\ Faculty of Electrical and \\ Electronic Eng. \\ University of Tun Hussein Onn \\ Malaysia, Johor, Malaysia
}

\author{
M. F. Ghanim \\ Dept. of Computer Eng. \\ College of Engineering \\ University of Mosul, Iraq
}

\begin{abstract}
The aim of this paper is to discuss the development of LTE algorithm which has the most advantages compare to other technologies such as WiMAX and WiFi. In order to improve LTE disadvantages is to increase the bandwidth at both sides (transmitter and receiver), where the current bandwidth is 100 $\mathrm{MHz}$ and the peak data rate is $1 \mathrm{Gpbs}$. The objective of this paper is to increase the bandwidth up to $100 \mathrm{MHz}$ through increasing the number of carrier aggregation in LTE system. This paper focus on the high-bandwidth internet access anytime, anywhere which is continuously increasing. As the spectrum is limited, this means mobile communications systems have to support larger bandwidth than today's systems. Peak data rates up to $1 \mathrm{Gbps}$ are expected from bandwidths of $100 \mathrm{MHz}$ where OFDM adds additional subcarrier to increase bandwidth. Unfortunately, the available bandwidth may not be continuous as a result of fragmented spectrum. In addition, this feature allows scalable expansion of effective bandwidth delivered to a user terminal through concurrent utilization of radio resources across multiple carriers. Therefore, in order to support bandwidths greater than $20 \mathrm{MHz}$, two or more component carriers (CCs) (of the same or different bandwidths) are aggregated together in LTE-A. This way, wider transmission bandwidths are supported in the downlink (DL) or uplink (UL) between User equipment (UE) and Evolved Node B (eNB).
\end{abstract}

\section{General Terms}

4G Technology, Telecommunication Engineering, Wireless.

\section{Keywords}

LTE-A; CA; CC; Intra band aggregation.

\section{INTRODUCTION}

The key part of LTE-Advanced is the Carrier Aggregation (CA). The principle with CA is to extend the maximum bandwidth in the uplink or downlink (or both) directions by aggregating multiple carriers. The carriers to be aggregated are basically Release 8 carriers, which then facilitate the necessary backwards compatibility.
Release 10 LTE-Advanced UE resource pool

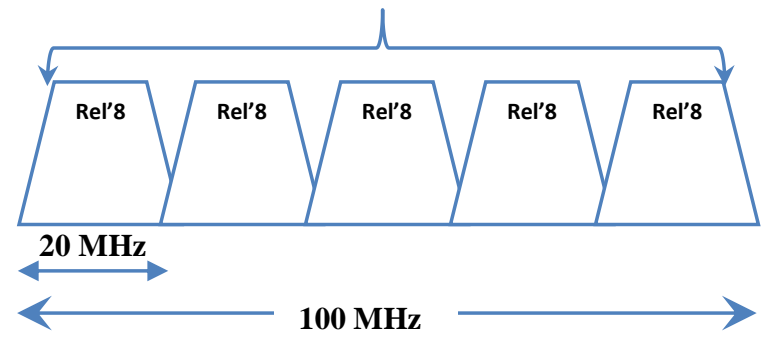

Fig 1: Mixing LTE-Advanced and legacy devices

A pre-Release 10 UE can access one of the component carriers while CA-capable UEs will then operate on multiple component carriers as shown in Figure 1[1]. Component carriers (CCs) other than the one intended for Release 8 devices could contain performance-enhancement methods not compatible with the pre-Release 10 UE. However the methods agreed so far for Release 10 are such that Release 8 and 9 UEs can also access the carrier even if they do not benefit from the added improvements. The LTE-Advanced CA can basically take place both within a frequency band and between frequency bands in 3GPP. It has been agreed that work will be done so that Release 10 will contain the following:

- $\quad$ Support for the downlink intra and inter-band CA, with the motivation that in most cases each operator does not have more than $20 \mathrm{MHz}$ in a given frequency band and thus inter-band carrier aggregation in the downlink direction is more easily usable.

- In the uplink direction, carrier aggregation is not seen to be attractive because the use of two transmitters simultaneously in the UE is more challenging than two receivers. The same approach has been used with HSPA where only the downlink multiband operation has been defined in Release 9 .

In Release 11, work is expected to continue on the uplink carrier aggregation between the bands. Release 10 will contain a limited set of frequency bands (and band combinations) where the downlink carrier aggregation has been defined, including UE performance and RF requirements. The bands to be considered will not be limited 
by the physical layer and signaling specifications but work on the necessary RF and performance studies will need to be done for a limited set of band combinations as quite a lot of work is required for each particular frequency band combination [2].

\section{CARRIER AGGREGATION IN LTE AND LTE-ADVANCED}

The possibility for carrier aggregation was introduced in LTE release 10 . In the case of carrier aggregation, multiple LTE carriers, each with a bandwidth up to $20 \mathrm{MHz}$, can be transmitted in parallel to/from the same terminal, thereby allowing for an overall wider bandwidth and correspondingly higher per-link data rates. In the context of carrier aggregation, each carrier is referred to as a component carrier $(C C)$ as, from an RF point-of-view; the entire set of aggregated carriers can be seen as a single (RF) carrier. The possibility of up to five component carriers, for different bandwidths of up to $20 \mathrm{MHz}$, can be aggregated allowing for an overall transmission bandwidths up to $100 \mathrm{MHz}$ [3]. A terminal capable of carrier aggregation may receive or transmit simultaneously on multiple component carriers. Each $\mathrm{CC}$ can also be accessed by an LTE terminal from the earlier releases that is, component carriers are backwards compatible [4].

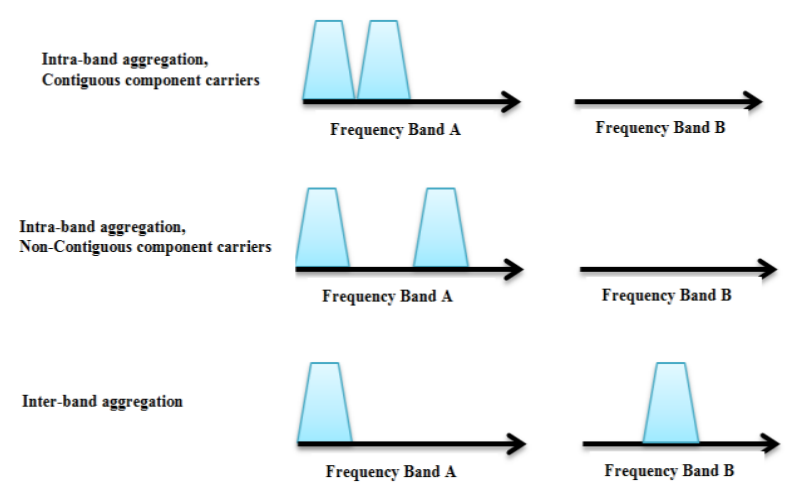

Fig 2: Different types of carrier aggregation [4]

As noted, that aggregated component carriers do not need to be contiguous in the frequency domain, but rather, with respect to the frequency location of the different component carriers. Three different cases can be identified as shown in Figure 2:

- Intra-band aggregation with frequency-contiguous component carriers

- Intra-band aggregation with non-contiguous component carriers

- Inter-band aggregation with non-contiguous component carriers

Carrier aggregation is supported for both Frequency Division Duplex (FDD) and Time Division Duplex (TDD), although all component carriers require to have the same duplex scheme. Furthermore, in the case of TDD, the uplink-downlink configuration should be the same across component carriers. The special subframe configuration can be different for different components carriers, as long as the resulting downlink-uplink switching time is sufficiently large [4].

\subsection{Intra-band aggregation with frequency-contiguous component carriers}

This is where a contiguous bandwidth wider than $20 \mathrm{MHz}$ is used for CA (Figure 2). Although this may be a less likely scenario given frequency allocations today, it can be common when new spectrum bands like $3.5 \mathrm{GHz}$ are allocated in the future in various parts of the world. The spacing between center frequencies of contiguously aggregated CCs is a multiple of $300 \mathrm{kHz}$ to be compatible with the $100 \mathrm{kHz}$ frequency raster of Release 8/9 and preserving orthogonally of the subcarriers with $15 \mathrm{kHz}$ spacing.

\subsection{Intra-band aggregation with non- contiguous component carriers}

This is where multiple CCs belonging to the same band are used in a non-contiguous manner (Figure 2). This scenario can be expected in countries where spectrum allocation is noncontiguous within a single band, when the middle carriers are loaded with other users, or when network sharing is considered. Therefore this model would fit operators in North America or Europe, who have fragmental spectrum in one band or share the same cellular network[5].

\subsection{Inter-band aggregation with non- contiguous component carriers}

Inter-Band Carrier Aggregation implies that carriers in different operating bands are aggregated (see also the last example in Figure 2). Many RF properties within a band can, to a large extent, remain the same as for a single carrier case. There is, however, impact for the UE, due to the possibility for inter modulation and cross-modulation within the UE device when multiple transmitter and receiver chains are operated simultaneously. For the base station it has very little impact, since in practice it corresponds to a base station supporting multiple bands, which is a configuration not really treated in RF specifications.

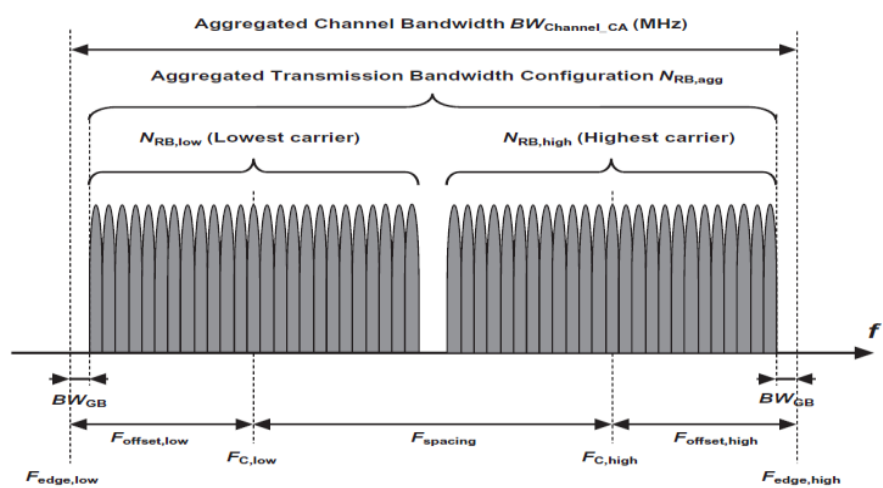

Fig 3: Definitions for Intra-Band Carrier Aggregation RF parameters, for example with two aggregated carriers [2]

For intra-band carrier aggregation, the definitions of $B W_{\text {channel }}$ and number of resource blocks (NRB) as shown in Figure 4 still apply for each component carrier, while new definitions are needed for the Aggregated Channel Bandwidth $\left(B W_{\text {Channel CA }_{C}}\right)$ and the Aggregated Transmission bandwidth Configuration ( $N \mathrm{RB}$,agg) as shown in Figure 3. In connection with this, a new capability is defined for the UE called Carrier Aggregation Bandwidth Class. 


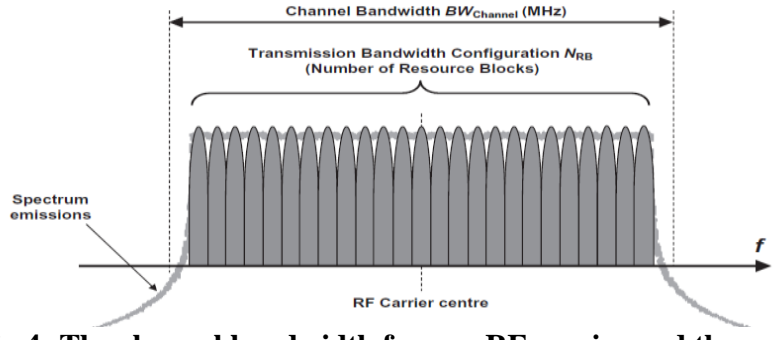

Fig 4: The channel bandwidth for one RF carrier and the corresponding transmission bandwidth configuration

For the specification, RF requirements are based on a nominal channel spacing that is derived from the channel bandwidth of

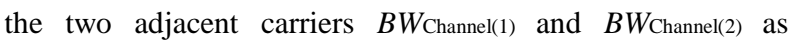
follows:

$F_{\text {Spacing_Nominal }}=\left|\frac{B W_{\text {Channel(1) }}+B W_{\text {Chamel(2) }}-0.1\left|B W_{\text {Channel(1) }}-B W_{\text {Channel(2) }}\right|}{2 \cdot 0.3}\right| 0.3$

In order to allow for a tighter packing of component carriers, the value of $F$ Spacing can be adjusted to any multiple of 300 $\mathrm{kHz}$ that is smaller than the nominal spacing, as long as the carriers do not overlap. RF requirements for LTE are normally defined relative to the channel bandwidth edges. For intraband CA, this is generalized so that requirements are defined relative to the edges of the Aggregated Channel Bandwidth, identified in Figure 3 as $F$ edge,low and $F$ edge,high. In this method many RF requirements can be reused, but with new reference points in the frequency domain. The aggregated channel bandwidth for both UE and base station is defined as follows:

$B W_{\text {Channel_CA }}=F_{\text {edge,high }}-\boldsymbol{F}_{\text {edge,low }}$

The location of the edges is defined relative to the carriers at the edges through a new parameter $F_{\text {offset }}$ (refer Figure 3 ) using the following relation to the carrier center positions $F_{C}$ of the lowest and highest carriers:

$$
\begin{aligned}
& F_{\text {edge,low }}=F_{C, \text { low }}-F_{\text {offset,low }} \\
& F_{\text {edge,high }}=F_{C, \text { high }}+F_{\text {offset,high }}
\end{aligned}
$$

The value of $F_{\text {offset }}$ for the edge carriers and the corresponding location of the edges are, however, not defined in the same way for UE and base station.

For the base station, there are legacy scenarios where the base station receives and transmits adjacent independent carriers, supporting legacy terminals of earlier releases using single carriers. This scenario will also have to be supported for a configuration of aggregated carriers. In addition, for backward compatibility reasons, a fundamental parameter such as channel bandwidth and the corresponding reference points (the channel edge) for all RF requirements will have to be remaining the same. The implication is that the channel edges shown in Figure 4 for each CC will also remain as reference points when the carriers are aggregated. This results in the following base station definition of $F_{\text {offset }}$, for carrier aggregation, which is "inherited" from the single carrier scenario:

$$
F_{\text {offset }}=\frac{B W_{\text {Channel }}}{2}\left(F_{0} r_{-} \text {base_station }\right)
$$

Unlike the base station, the UE is not restricted by legacy operation, but rather from the nonlinear properties of the PA and the resulting is an unwanted emissions mask. At both edges of the aggregated channel bandwidth, a guard band $B W_{\mathrm{GB}}$ will be required, in order for the emissions to reach a level where the out-of-band emissions limits in terms of an emission mask are applied. Whether a single wide carrier or multiple aggregated carriers of the same or different sizes are transmitted, the guard band required will have to be the same at both edges, since the emission mask roll-off is the same. A problem with the backwards-compatible base station definition is that the resulting guard $B W_{\mathrm{GB}}$ is proportional to the channel BW and would therefore be different if carriers of different channel BW are aggregated.

For this reason, a different definition is used for the UE, based on a "symmetrical" guard band. For the edge carriers (low and high), $\quad F_{\text {offset }}$ is half of the transmission bandwidth configuration, plus a symmetrical guard band $B W_{\mathrm{GB}}$ :

$$
F_{\text {offset }}=\frac{0.18 \mathrm{MHz} \cdot N_{R B}}{2}+B W_{G B} \operatorname{For}(U E)
$$

Where $0.18 \mathrm{MHz}$ is the bandwidth of one resource block and $B W_{\mathrm{GB}}$ is proportional to the channel $\mathrm{BW}$ of the largest component carrier. For the CA bandwidth classes defined in Release 10, where the edge carriers have the same channel bandwidth, $F_{\text {offset }}$ will be the same for terminals, base stations and $B W$ Channel_CA will also be the same.

It may look like an anomaly that the definitions may potentially lead to slightly different aggregated channel BW for the UE and the base station, but this is in fact not a problem. UE and base station requirements are defined separately and do not have to cover the same frequency ranges. The aggregated channel $\mathrm{BW}$ for both UE and base station do, however, have to be within an operator's license block in the operating band. Once the frequency reference point is set, the actual RF requirements are to a large extent the same for a single carrier configuration.

\section{COMPONENT CARRIERS - AGGREGATION}

Carrier aggregation, where two or more component carriers are aggregated, is considered for LTE-Advanced, in order to support wider transmission bandwidths, for example, up to $100 \mathrm{MHz}$ and also for spectrum aggregation. The principle of carrier aggregation of four component carriers is shown in Figure 5. The physical layer specification shall support carrier aggregation for both contiguous and non-contiguous component carriers. Both cases are covered in the example depicted in Figure 5 [6]. 


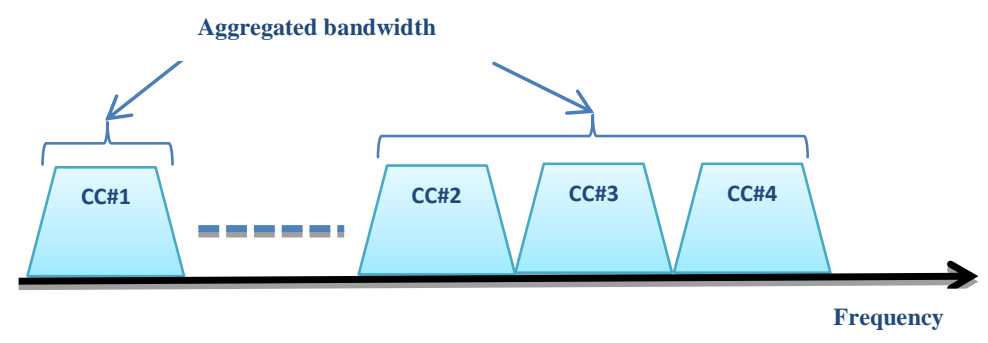

Fig 5: Carrier aggregation principles

DL multiple access scheme (waveform) supporting wider bandwidth is $\mathrm{N} \times$ OFDMA [7], [8]. This means that LTEAdvanced DL continues on the Orthogonal Frequency Division Multiple Access (OFDMA) track. The reason for selecting N x OFDMA is that it will provide full support for component carrier specific modulation and coding scheme (MCS), Hybrid ARQ (HARQ) and power control. The selected approach provides also the best backwards compatibility between LTE Rel-8 and enables that majority of existing control signaling can be directly borrowed from LTE Rel-8. The block diagram for considering transmitter is shown in Figure 6 [9]. There is one transport block per component carrier (in the case without spatial multiplexing). The transport block is turbo-encoded and modulated and after the modulator. The multiple Inverse Fast Fourier Transform (IFFTs) shown in the figure may be replaced by one large IFFT on the condition that the component carriers are located on the common $15 \mathrm{kHz}$ raster. It is also noted that support for multiple DL component carriers is an eNodeB category feature.

Besides the multi-carrier extension it has also been decided to support clustered OFDMA (non-contiguous pseudorandom binary sequence (PRB) allocation) within each component carrier. Furthermore, it has been decided that on top of Rel-8 operation, control-data decoupling (simultaneous Physical Downlink Control Channel (PDCCH) and Physical Downlink Shared Channel (PDSCH) transmission) is also supported.

In conclusion it is noted that the possibility for low- Cubic Metric (CM) transmission is fully maintained in LTEAdvanced including Single User-Multi Input Multi Output (SU-MIMO). The additional options increasing the CM compared to Rel- 8 are designed to enable higher bandwidth (CC aggregation), increased scheduling flexibility (clustered OFDMA) and data-control separation. Furthermore, the new features are fully backwards compatible and can be design with rather minor standardization efforts.

\section{CARRIER AGGREGATION PERFORMANCE}

The use of carrier aggregation benefits system performance in three ways:

- There is an increased peak data rate when enabling the aggregation of spectra for more than single frequency band. The theoretical peak data rate from the combination use of carrier aggregation with total of $40 \mathrm{MHz}$ spectrum and up to eight antennas reaches up to $1 \mathrm{Gbps}$ in the downlink and in the uplink up to $500 \mathrm{Mbps}$ with the technologies.

- Improved average user throughput, especially when the number of users is not too high. Joint carrier scheduling in the eNodeB allows the optimal selection of the carrier to use thus leading to better performance and optimal load balancing between the carriers.

\section{SIMULATION AND RESULTS}

The program SystemVue (product of Agilent Company) is used to clarify the types of Intra band Contiguous Component Carriers on LTE-Advanced. The results show the CA Spectrum Power simulation for different bandwidths of LTEAdvanced System (40 MHz, $60 \mathrm{MHz}, 80 \mathrm{MHz}$, and 100 $\mathrm{MHz}$ ) [10].

\section{LTE-ADVANCED DL INTRA BAND CONTIGUOUS CARRIER} AGGREGATION

The following simulation is to generate LTE-Advanced downlink signals with carrier aggregation. The frequency band (center frequency), bandwidth of the component carrier, oversampling ratio and the number of Transmitter antennas can be changed in the parameter tab.

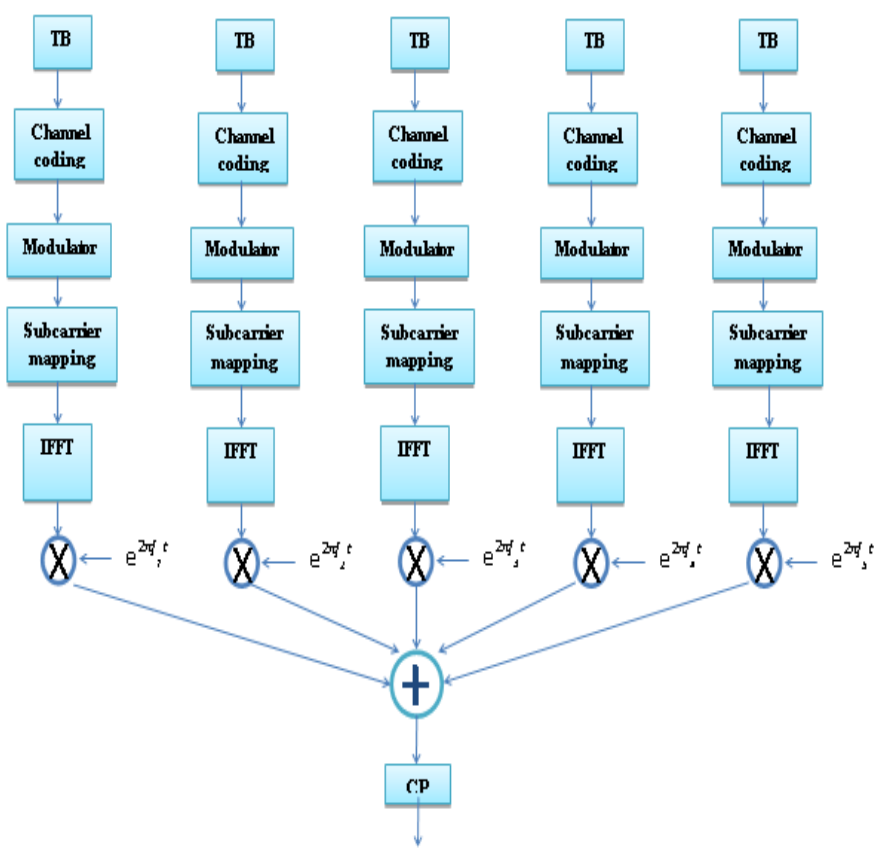

Fig 6: Block diagram for N x OFDMA

\subsection{LTE-Advanced Downlink Contiguous Carrier Component to support $40 \mathrm{MHz}$}

Generation of $40 \mathrm{MHz}$ LTE-Advanced contiguous carrier aggregation (Two $20 \mathrm{MHz}$ component carriers) is simulated. Figure 7 shows the downloading of the $40 \mathrm{MHz}$ LTE-A DL signal. 


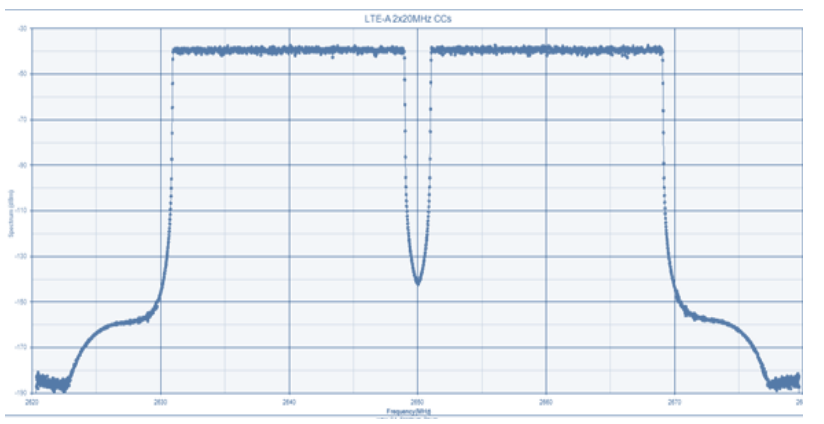

Fig 7: LTE-A with bandwidth $40 \mathrm{MHz}$

\subsection{LTE-Advanced Downlink Contiguous Carrier Component to support $60 \mathrm{MHz}$}

Generation of $60 \mathrm{MHz}$ LTE-Advanced contiguous carrier aggregation (Three $20 \mathrm{MHz}$ component carriers) is simulated. Figure 8 shows the downloading of the $60 \mathrm{MHz}$ LTE-A DL signal.

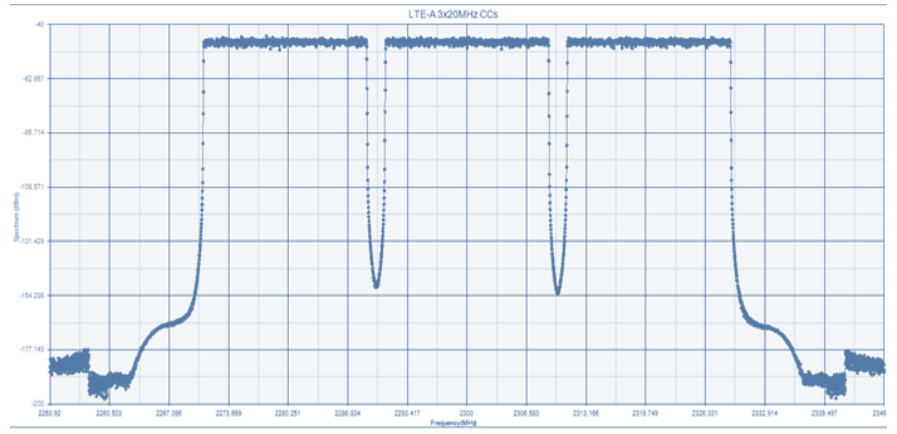

Fig 8: LTE-A with bandwidth $60 \mathrm{MHz}$

\subsection{LTE-Advanced Downlink Contiguous Carrier Component to support $80 \mathrm{MHz}$}

Generation of $80 \mathrm{MHz}$ LTE-Advanced contiguous carrier aggregation (Four $20 \mathrm{MHz}$ component carriers) is simulated. Figure 9 shows the downloading of the $80 \mathrm{MHz}$ LTE-A DL signal.

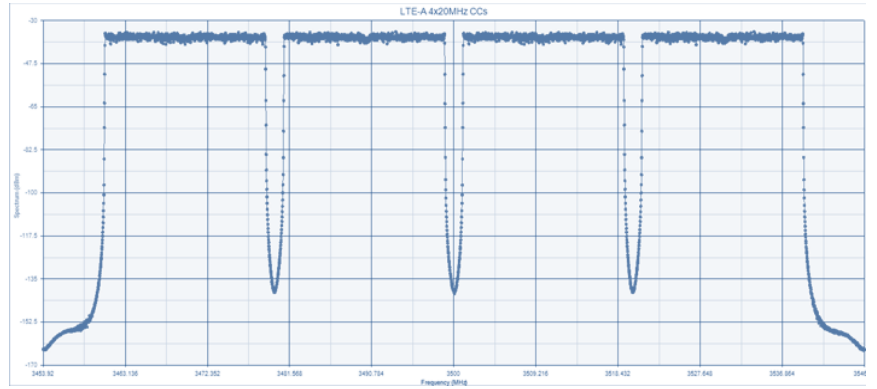

Fig 9: LTE-A with bandwidth $80 \mathrm{MHz}$

\subsection{LTE-Advanced Downlink Contiguous Carrier Component to support $100 \mathrm{MHz}$}

Generation of $100 \mathrm{MHz}$ LTE-Advanced contiguous carrier aggregation (five $20 \mathrm{MHz}$ component carriers) is simulated. Figure 10 shows the downloading of the $100 \mathrm{MHz}$ LTE-A DL signal.

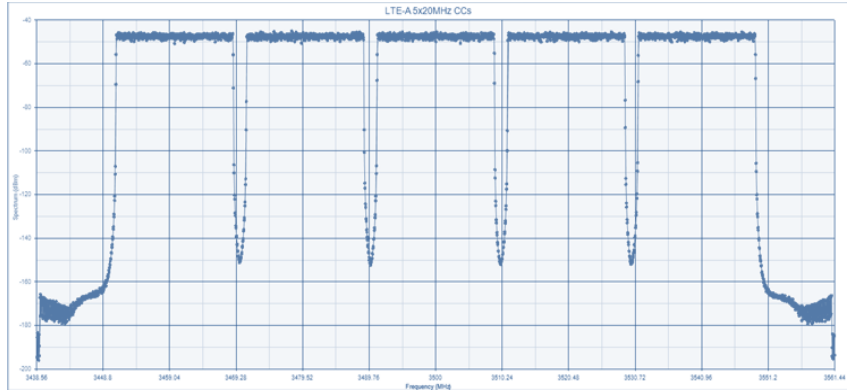

Fig 10: LTE-A with bandwidth $100 \mathrm{MHz}$

Therefore the result from Figure 7 to Figure 10 can be summarizes in the following Figure 11. The figure shows the effect of increasing the number of component carriers on the bandwidth of LTE-Advanced system.

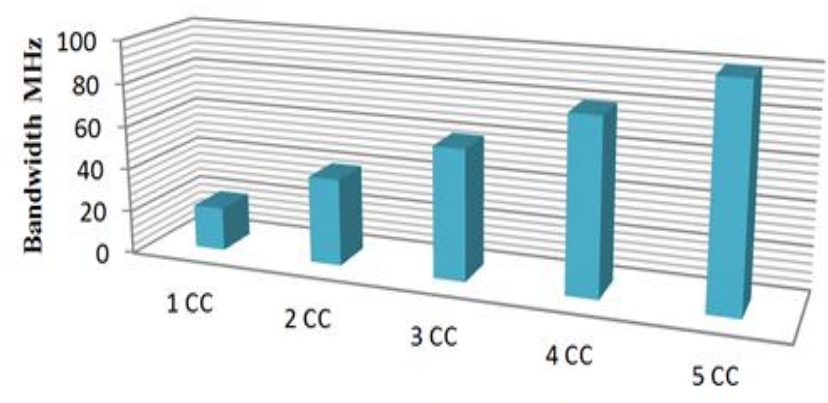

No. of Component Carriers

Fig 11: Relationship between component carriers and bandwidth MHz

\section{CONCLUSION}

This paper focuses on the different types of carrier aggregation which contains the intra-band carrier aggregation (contiguous and non-contiguous) and inter-band contiguous carrier aggregation.

The main target for using carrier aggregation in this work is to achieve up to $1 \mathrm{Gbps}$ peak data rate in the downlink and 500 Mbps in the uplink over wide frequency bandwidths (up to $100 \mathrm{MHz}$ ) in the new LTE-Advanced standards.

The simulation for bandwidth equal to $40,60,80$ and 100 $\mathrm{MHz}$ is perform using the program SystemVue (product of Agilent Company), these cases are simulated for intra-band contiguous carrier component in downlink and the results show that increasing the number of carrier components is directly proportional with the bandwidth and peak data rate. The simulation result also shows that best performance for LTE-Advanced are achieved when five carrier components are used. In this case the bandwidth of the channel is equal to $100 \mathrm{MHz}$ and the peak data rate reaches $1 \mathrm{Gbps}$.

\section{ACKNOWLEDGMENT}

This research is funded by the Postgraduate Incentive Grant, University Tun Hussein Onn Malasyia (Vot 0894). The authors are grateful to Faculty of Electrical and Electronic Engineering for the technical support in carrying out this study. 


\section{REFERENCES}

[1] Y. Wang, Klaus I. Pedersen, Troels B. Sørensen and Preben E. Mogensen. 2010. Carrier Load Balancing and Packet Scheduling for Multi-Carrier Systems", IEEE transactions on wireless communications Vol. 9, NO. 5, (May 2010), 1780.

[2] H. Holma and A. Toskala. 2011 LTE for UMTS Evolution to LTE-Advanced Second Edition, Wiley, UK, 489-491.

[3] Ghosh, A.; Ratasuk, R.; Mondal, B.; Mangalvedhe, N., and Thomas, T.; 2010 LTE-ADVANCED: nextgeneration wireless broadband technology, IEEE Wireless Communications Vol.17, issue 3, (June 2010), 13.

[4] Dahlman, E., Parkvall S., and Sköld J. 2011 4G LTE/LTE-Advanced for Mobile Broadband", Elsevier UK, 132-134.

[5] Iwamura, M.; Etemad, K.; Mo-Han Fong; Nory, R.; Love, R. 2010 Carrier Aggregation Framework in 3GPP
LTE-Advanced, IEEE Communications Magazine, Vol. 48, Issue 8 (Aug. 2010), 61

[6] Krouk E., and Semenov S. 2011 modulation and coding techniques in wireless communications, Wiley, UK, 600603.

[7] R1-081842, LTE-A Proposals for evolution, Nokia Siemens Networks, Nokia.

[8] R1-083732, Comparison between SC-FDMA and OFDMA for LTE-Advanced Uplink, Nokia Siemens Networks, Nokia.

[9] R1-082609, Uplink Multiple access for LTE-Advanced Nokia Siemens Networks, Nokia Siemens Networks, Nokia.

[10] Agilent EEsof EDA, Jinbiao Xu. 2011 LTE-Advanced Signal Generation and Measurement Using SystemVue, Agilent Technologies. 